\title{
DECAIMENTO DA TURBULÊNCIA CONVECTIVA: UMA ESTIMATIVA DO COEFICIENTE DE DIFUSÃO TURBULENTO NA CAMADA RESIDUAL
}

\author{
A. Goulart \\ Dep. de Ciências Exatas \\ Universidade Regional Integrada - S. Ângelo, RS \\ G. Degrazia \\ Departamento de Física, CCNE \\ UFSM, Santa Maria, RS \\ D. Anfossi \\ CNR - Istituto di Cosmogeofísica \\ Turin, Itália \\ H. F. de Campos Velho \\ Instituto Nacional de Pesquisas Espaciais - LAC
}

\section{RESUMO}

Neste trabalho é apresentado um modelo que descreve o decaimento da energia cinética turbulenta convectiva e estima o coeficiente de difusão turbulento na Camada Residual. A equação dinâmica para a função espectro de energia é obtida da forma clássica a partir da equação de Navier-Stokes. São considerados os termos que descrevem a transferência inercial de energia, a produção ou destruição de energia por efeito térmico e a dissipação molecular. A transferência inercial de energia é parametrizada a partir da teoria estatística de Taylor. Emprega-se a teoria de Heisenberg 
baseada no conceito de uma viscosidade cinemática turbulenta para descrever a transferência inercial de energia dos grandes para os pequenos turbilhões. O termo que descreve a produção ou destruição de energia cinética por efeito térmico é parametrizado a partir da teoria de similaridade convectiva considerando-se a hipótese de Pao, a qual supõe que a energia é extraída do fluxo médio de forma contínua, o que permite não explicitar uma escala de tempo característica. Pelo fato das equações dinâmicas que descrevem o fluxo turbulento serem válidas somente no espaço tridimensional, foi obtido um espectro 3-D da Camada Convectiva a partir de um modelo proposto por Kristensen, e do modelo para os espectros unidimensionais obtido por Degrazia. O coeficiente de difusão turbulento é determinado a partir da teoria estatística de Taylor e teoria de similaridade convectiva. Os resultados obtidos neste trabalho são comparados com o modelo de LES.

\section{SUMMARY}

In this work is presented a model that describes the decay of the convective turbulent kinetic energy and that estimates the turbulent diffusion coefficient in the Residual Layer. The dynamic equation for energy spectrum function is obtained from Navier-Stokes equation. The terms that describe the inertial transfer of energy, the production or destruction of energy for thermal effect and the molecular dissipation are considered. The inertial transfer of energy is calculated from the Taylor's statistical diffusion theory. The Heisenberg's theory that is based on the concept of a kinematic turbulence viscosity to describe the inertial transfer of energy from the big to the small eddies is used. The term that describes the production or destruction of kinetic energy for thermal effect is obtained from the convective similarity theory considering the Pao's hypothesis. This hypothesis supposes that energy is extracted of the medium flow in a continuous way, allowing do 
not explicit a scale of time characteristic. The dynamic equations that describe the turbulent flow are only valid in the three-dimensional space. For this reason it was obtained one spectrum 3-D for convective layer from a model proposed by Kristensen, and of a model for the one-dimensional spectra calculated by Degrazia. The turbulent diffusion coefficient is obtained from the Taylor's statistical and the similarity convective theories. The results obtained in this work are compared with the LES model.

\section{INTRODUÇÃO}

Nas últimas décadas houve um grande avanço na compreensão da dispersão de poluentes na Camada Limite Convectiva (CLC) e Camada Limite Estável (CLE). Entretanto existem poucos trabalhos envolvendo a Camada Residual (CR). Aproximadamente meia hora antes do pôr-do-sol o fluxo de calor do solo cessa e começa um processo de decaimento do fluxo turbulento que forma a camada convectiva. A camada de ar resultante é denominada Camada Residual devido as suas variáveis de estado e concentração iniciais serem as mesmas da camada convectiva previamente existente. A CR não tem contato direto com a superfície terrestre, porém tem sua base modificada pelo avanço da camada estável durante o decorrer da noite. Assim, o restante da CR não é afetado pelo transporte turbulento e propriedades relacionadas com a superfície (Stull, 1988).

O decaimento dos turbilhões que contém a energia cinética e a produção térmica de energia são os mecanismos físicos que podem manter eficiente o processo de dispersão na $\mathrm{CR}$. Além destes, a produção mecânica de turbulência pode estar presente na CR (Kim e Mahrt, 1992). Entretanto este mecanismo não será considerado neste trabalho. 
O objetivo básico do presente trabalho é propor um método semi-empírico para derivar o coeficiente de difusão no decaimento da turbulência, e aplicá-lo para o decaimento da turbulência convectiva na CR.

As expressões para o coeficiente de difusão derivadas neste trabalho podem ser usadas em modelos Eulerianos e Lagrangeanos de dispersão como também em modelos de mesoescala .

\section{DECAIMENTO DA ENERGIA CINÉtICA NA CAMADA LIMITE PLANETÁRIA}

Uma equação para a função espectro de energia em um fluxo turbulento pode ser derivada a partir de um dos princípios fundamentais da física, que é o princípio da conservação de momento, expresso pela equação de Navier-Stokes. Para uma turbulência homogênea obtemos (Hinze, 1975),

$$
\frac{\partial}{\partial t} \mathbf{E}(k, z, t)=\mathbf{M}(k, z, t)+\mathbf{W}(k, z, t)+\frac{g}{T_{0}} \mathbf{H}(k, z, t)-2 v k^{2} \mathbf{E}(k, z, t)
$$

onde $t$ é o tempo; $g$ é a aceleração da gravidade; $T_{o}$ é a temperatura absoluta na superfície; $k$ é o número de onda; $v$ é a viscosidade cinemática; $\mathbf{E}(k, z, t)$ é a função espectro de energia; $\mathbf{M}(k, z, t)$ é a produção de energia por efeito mecânico; $\mathbf{W}(k, z, t)$ descreve a transferência de energia, por efeito inercial, dos grandes para os pequenos turbilhões, $\mathbf{H}(k, z, t)$ é um termo de produção ou consumo de energia por efeito térmico (convecção) e o último termo representa a dissipação de energia pela viscosidade molecular.

Vamos considerar um fluxo turbulento homogêneo, em que o termo de produção mecânica possa ser desprezado. Nestas condições a equação (1) pode ser escrita como,

$$
\frac{\partial}{\partial t} \mathbf{E}(k, z, t)=\mathbf{W}(k, z, t)+\frac{g}{T_{o}} \mathbf{H}(k, z, t)-2 v k^{2} \mathbf{E}(k, z, t)
$$


Para parametrizar o termo de transferência de energia por efeito inercial $\mathbf{W}(k, z, t)$, será utilizada a teoria de Heisenberg (Degrazia et al., 1998), baseada no conceito de uma viscosidade cinemática turbulenta para descrever a transferência de energia cinética dos grandes para os pequenos turbilhões. De acordo com Heisenberg podemos escrever,

$$
\mathbf{W}(k, z, t)=-2 v_{T} k^{2} \mathbf{E}(k, z, t)
$$

onde $v_{T}$ é denominado coeficiente de viscosidade cinemático turbulento.

A escolha correta de $v_{T}$ é a maior dificuldade na hipótese de Heisenberg. Do ponto de vista físico a introdução do coeficiente de viscosidade cinemático para estimar a transferência de energia de grande para pequena escala pode ser correta somente se os pequenos turbilhões são estatisticamente independentes dos grandes turbilhões (Batchelor, 1959).

Em um fluxo turbulento geofísico como o existente na CLC, o número de Reynolds é muito grande e como conseqüência o espectro de energia pode ser subdividido em três regiões distintas: o subintervalo que contém a principal energia cinética, o subintervalo inercial e o subintervalo de dissipação (Hinze, 1975). No subintervalo que contém a principal energia cinética encontram-se os turbilhões que mais contribuem para a energia cinética turbulenta. O espectro neste intervalo apresenta um máximo. Podemos associar um número de onda $k_{e}$ a esse máximo para caracterizar os turbilhões desse subintervalo. No subintervalo de dissipação, por efeito da viscosidade, podemos associar um número de onda $k_{d}$ ao máximo do espectro. Este valor de $k_{d}$ caracteriza os turbilhões pertencentes a este subintervalo.

Para um número de Reynolds elevado $(v \rightarrow 0)$ espera-se que a dissipação, na região de número de onda muito abaixo da região de máxima dissipação, seja negligenciável quando comparada com o fluxo de energia transferido por efeito inercial. Em tal subintervalo o efeito da viscosidade 
molecular desaparece $v_{T} \gg>v$, e como conseqüência podemos associar um número de onda $k_{I}$ com a escala dos turbilhões que fornecem a contribuição principal para este fluxo de energia inercial, de forma que

$$
k_{e}<<k_{I}<<k_{d} \text {. }
$$

Com a condição (4) podemos supor que a turbulência neste intervalo é estatisticamente independente do subintervalo que contém a principal energia cinética e do subintervalo de forte dissipação. Esta região do espectro é denominada de subintervalo inercial. O subintervalo inercial satisfaz a condição de independência estatística dos turbilhões, necessária para a validade de uma expressão que descreva o coeficiente de viscosidade cinemático $v_{T}$.

O coeficiente de viscosidade cinemático turbulento pode ser calculado diretamente a partir da teoria estatística de Taylor para grandes tempos de viagem (Batchelor, 1949; Hinze, 1975; Hanna, 1981; Degrazia e Moraes, 1992; Degrazia et al., 1998)

$$
v_{T}=\frac{\beta}{6} \frac{\sigma^{2}}{n_{I}}
$$

onde $\beta$ é definido como a razão entre as escalas de tempo Lagrangeana e Euleriana; $n_{I}$ é a freqüência associada ao máximo do espectro no subintervalo inercial, dada em Hertz e $\sigma^{2}$ é a variância da velocidade turbulenta no subintervalo inercial.

Para parametrizar o termo que descreve a produção ou destruição por efeito térmico vamos considerar a hipótese de Pao (1965), a qual supõe que a energia é extraída do fluxo médio de forma contínua, o que permite não explicitar uma escala de tempo característica. Podemos escrever a seguinte expressão matemática para o termo de produção de energia térmica, 


$$
\frac{g}{T_{o}} \mathbf{H}(k, z, t)=\frac{g}{T_{o}} \frac{\partial \theta}{\partial z} c_{1} \varepsilon_{o}^{-1 / 3} k^{-2 / 3} \mathbf{E}(k, z, t)
$$

onde $\theta$ é a temperatura potencial, $\varepsilon$ é a razão de dissipação de energia e $c_{1}$ é uma constante a ser determinada.

Da definição de escala de velocidade convectiva podemos escrever

$$
\frac{g}{T_{o}}=\frac{w_{*}^{3}}{(\overline{w \theta})_{o} h}
$$

onde $(\overline{w \theta})_{0}$ é o fluxo de calor na superfície; $w_{*}$ é a escala de velocidade no regime de estabilidade convectivo; $h$ é a altura da camada convectiva.

A partir do modelo de LES (Large Eddy Simulation) desenvolvido por Nieuwstadt e Brost em 1986 podemos escrever,

$$
w_{*}^{2}=\frac{1}{2} \frac{\sigma_{w}^{2}}{f\left(t_{*}\right)}
$$

onde $\sigma_{w}^{2}$ é a variância da componente vertical da velocidade, $t_{*}=\frac{w_{*} t}{h}$ é um tempo adimensional e,

$$
f\left(t_{*}\right)=\frac{0,13}{1+1,5 t_{*}^{2}}
$$

Substituindo (8) em (7) obtemos,

$$
\frac{g}{T_{o}} \frac{\partial \theta}{\partial z}=\frac{1}{2} \frac{w_{*}}{f\left(t_{*}\right) h(\overline{w \theta})_{o}} \sigma_{w}^{2} \frac{\partial \theta}{\partial z}
$$

Ainda considerando o trabalho de Nieuwstadt e Brost (1986), podemos escrever,

$$
\sigma_{w}^{2} \frac{\partial \theta}{\partial z}=(\overline{w \theta})_{o} \frac{w_{*}}{h} F\left(t_{*}\right)
$$

onde $F\left(t_{*}\right)$ é uma função adimensional dada por 


$$
F\left(t_{*}\right)=70 \frac{t_{*}^{3}}{\exp \left(5 t_{*}\right)}+0,08
$$

Substituindo (11) em (10) temos,

$$
\frac{g}{T_{o}} \frac{\partial \theta}{\partial z}=\frac{1}{2}\left(\frac{w_{*}}{h}\right)^{2} \frac{F\left(t_{*}\right)}{f\left(t_{*}\right)}
$$

Este termo descreve o decaimento, porém não considera o estado inicial. De acordo com Moeng (Moeng et al, 1994) podemos escrever

$$
\frac{g}{T_{o}}(\overline{w \theta})=\frac{w_{*}^{3}}{h}\left(1-1,2 \frac{z}{h}\right)
$$

Substituindo (13) em (6), e considerando o estado inicial dado por (14), podemos escrever,

$$
\begin{aligned}
\frac{g}{T_{o}} \mathbf{H}(k, z, t) & =\frac{w_{*}^{3}}{h}\left(1-1,2 \frac{z}{h}\right)+ \\
& -\frac{1}{2}\left(\frac{w_{*}}{h}\right)^{2}\left(\frac{F\left(t_{*}\right)}{f\left(t_{*}\right)}-\frac{F(0)}{f(0)} \delta\left(t_{*}-0\right)\right) c_{1} \varepsilon_{o}^{-1 / 3} k^{-2 / 3} \mathbf{E}(k, z, t)
\end{aligned}
$$

onde $z$ é a altura a partir da superfície. A função delta de Dirac foi utilizada para que a equação (15) obedeça a lei de conservação de energia.

De acordo com o modelo de LES o gradiente de temperatura é constante e igual a $2 \times 10^{-4^{\circ}} \mathrm{Cm}^{-1}$ a partir de $t_{*} \approx 1$. Logo é possível determinar o termo de produção ou destruição de energia diretamente pela equação (6). Isto será feito a partir de $t_{*}=4$, quando o fluxo de calor tornase estável (Nieuwstadt et al, 1986).

$$
\begin{aligned}
& \text { Portanto para } t_{*}>4 \text { temos, } \\
& \frac{g}{T_{o}} \mathbf{H}\left(k, z, t_{*}\right)=\frac{w_{*}^{3}}{\left(\frac{w}{w \theta}\right)_{o} h} \frac{\partial \theta}{\partial z} c_{2} \varepsilon_{o}^{-1 / 3} k^{-2 / 3} \mathbf{E}\left(k, z, t_{*}\right)
\end{aligned}
$$

onde $c_{2}$ é uma constante a ser determinada a partir de dados experimentais ou de simulação numérica.

70 Rev. Ciência e Natura, Dispersion Process: 63 - 78 , 2000. 
Substituindo (3), (15) e (16) na equação (2) obtemos,

$$
\begin{aligned}
\frac{\partial \mathbf{E}(k, z, t)}{\partial t} & =\frac{w_{*}^{3}}{h}\left(1-1,2 \frac{z}{h}\right)+ \\
& -\frac{1}{2}\left(\frac{w_{*}}{h}\right)^{2}\left(\frac{F\left(t_{*}\right)}{f\left(t_{*}\right)}-\frac{F(0)}{f(0)} \delta\left(t_{*}-0\right)\right) c_{1} \varepsilon_{o}^{-1 / 3} k^{-2 / 3} \mathbf{E}(k, z, t) H_{e}\left(4-t_{*}\right)+ \\
& +\frac{w_{*}^{3}}{\left(\frac{w \theta}{w \theta}\right)_{o} h} \frac{\partial \theta}{\partial z} H_{e}\left(t_{*}-4\right) c_{2} \varepsilon_{o}^{-1 / 3} k^{-2 / 3} \mathbf{E}(k, z, t)-2 v_{T} k^{2} \mathbf{E}(k, z, t)
\end{aligned}
$$

onde foi considerado que $v_{T} \gg v$ (Degrazia et al, 1998) e $H_{e}$ é a função de Heaviside, definida por

$$
H_{e}(x)=\left\{\begin{array}{lll}
0 & \text { se } & x<0 \\
1 & \text { se } & x>0
\end{array}\right\}
$$

Em $x=0$ foi definido $H_{e}(0) \equiv 1$.

Vamos considerar as seguintes grandezas adimensionais,

$$
t_{*}=\frac{w_{*} t}{h} ; \quad R=\frac{w_{*} h}{v_{T}} ; \quad \varepsilon=\frac{\psi_{\varepsilon} w_{*}^{3}}{h}
$$

onde $\psi_{\varepsilon}$ é a função razão de dissipação adimensional.

Substituindo (19) em (17) temos a seguinte equação dinâmica para o espectro de energia no decaimento da turbulência convectiva,

$$
\frac{\partial \mathbf{E}\left(k, z, t_{*}\right)}{\partial t_{*}}+P\left(k, t_{*}\right) \mathbf{E}\left(k, z, t_{*}\right)=H^{\prime}
$$

onde 


$$
\begin{gathered}
P\left(k, t_{*}\right)=\frac{1}{2}\left(\frac{F\left(t_{*}\right)}{f\left(t_{*}\right)}-\frac{F(0)}{f(0)} \delta\left(t_{*}-0\right)\right) c_{1} \psi_{\varepsilon}^{-1 / 3}(k h)^{-2 / 3} H_{e}\left(4-t_{*}\right)+ \\
-\frac{w_{*} h}{\left(\frac{w \theta}{w \theta}\right)_{o}} \frac{\partial \theta}{\partial z} c_{2} \psi_{\varepsilon}^{-1 / 3}(k h)^{-2 / 3} H_{e}\left(t_{*}-4\right)+\frac{2(k h)^{2}}{R} \\
H^{\prime}=w_{*}^{2}\left(1-1,2 \frac{z}{h}\right) H_{e}\left(4.01-t_{*}\right)
\end{gathered}
$$

A evolução no tempo do espectro de energia durante o decaimento da turbulência convectiva é dada pela solução da equação (20),

$$
\mathbf{E}\left(k, z, t_{*}\right)=\left(\int_{0}^{t_{*}} H^{\prime} \exp \left(\int_{0}^{t^{\prime}} P(k, t) d t\right) d t^{\prime}+E_{o}(k, z)\right) \exp \left(-\int_{0}^{t_{t}} P(k, t) d t\right)
$$

Pelo fato de as equações dinâmicas que descrevem o fluxo turbulento serem válidas somente no espaço tridimensional o espectro inicial $E_{o}(k, z)$ que aparece na equação (23) é o espectro 3-D da Camada Convectiva. Para obter $E_{o}(k, z)$ vamos considerar o modelo de Kristensen (Kristensen et al, 1985) que determina o espectro tridimensional a partir dos espectros unidimensionais obtidos experimentalmente.

$$
E_{o}(k, z)=k^{3} \frac{d}{d k}\left(\frac{1}{k} \frac{d E_{u}}{d k}\right)+2 k^{4} \int_{0}^{1 / k^{2}} s^{2} g^{\prime \prime \prime}(s) d s-\frac{14}{9} k^{4 / 3} \int_{0}^{1 / k^{2}} s^{2 / 3} g^{\prime \prime \prime}(s) d s
$$

onde

$$
g^{\prime \prime \prime}(s)=2 f_{o u}^{\prime \prime \prime}(s)-f_{o v}^{\prime \prime \prime}(s)-f_{o w}^{\prime \prime \prime}(s)
$$

com

$$
f_{0 i}(s)=s^{2} E_{o i}(k) \quad i=u, v, w
$$

Para os $E_{o i}(k)$ serão usados os espectros unidimensionais de Degrazia (Degrazia et al, 1999), 


$$
E_{o i}(k)=\frac{\frac{1,06}{2 \pi} c_{i}\left(\frac{z}{h}\right)^{5 / 3} h \psi_{\varepsilon}^{2 / 3} w_{*}^{2}}{\left[\left(f_{m}^{*}\right)_{i}^{c}\right]^{j / 3}\left(1+\frac{1,5}{2 \pi} \frac{z}{h} h \frac{1}{\left(f_{m}^{*}\right)_{i}^{c}} k\right)^{5 / 3}}
$$

onde

$$
\begin{aligned}
& \psi_{\varepsilon}=\varepsilon h / w_{*}^{3}, w_{*}=\left(u_{*}\right)_{o}\left(-\frac{h}{\kappa L}\right)^{1 / 5},\left(f_{m}^{*}\right)_{i}^{c}=\frac{z}{B_{i} h}, B_{u}=B_{v}=1,5, \\
& B_{w}=1,8[1-\exp (-4 z / h)-0,0003 \exp (8 z / h)]
\end{aligned}
$$

A figura abaixo mostra o espectro 3-D da Camada Convectiva dado pela equação (24) com os espectros unidimensionais dados pelo modelo de Kristensen (Kristensen et al, 1985) e os espectros unidimensionais dados pela equação (27), (Degrazia et al, 1999). Podemos observar que os espectros unidimensionais de Degrazia podem ser utilizados no modelo de Kristensen (Kristensen et al, 1985) para se obter o espectro tridimensional da Camada Convectiva.

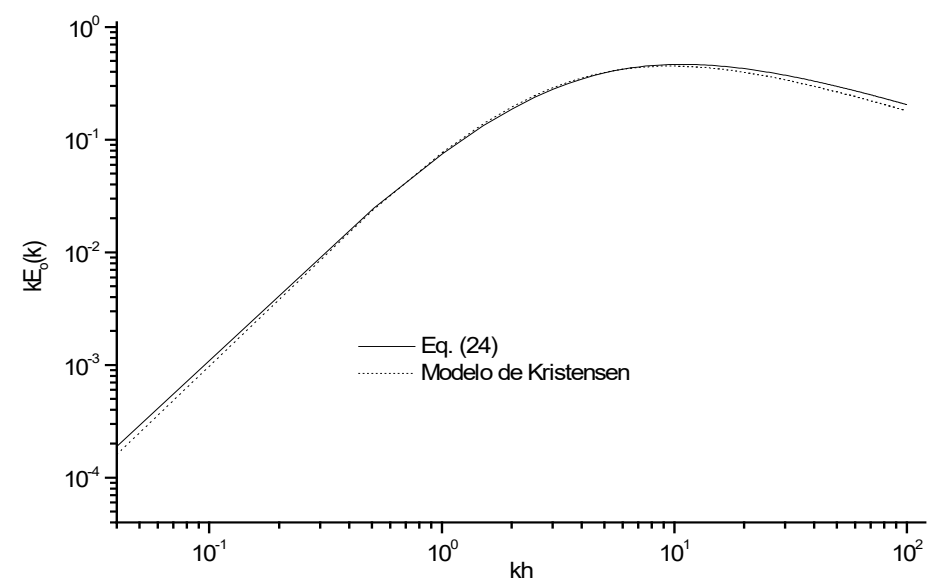

Fig.1 Espectro 3-D da CLC dado pela Eq. (24) com os espectros unidimensionais de Kristensen e dados pela Eq. (27) 
A partir da teoria estatística de Taylor, para grandes tempos de viagem, podemos determinar uma expressão para o coeficiente de difusão (Batchelor, 1949; Hinze, 1975; Hanna, 1981; Degrazia e Moraes, 1992; Degrazia et al., 1998),

$$
K(z, t)=\frac{\beta}{6} \frac{\sigma^{2}(z, t)}{n_{e}}
$$

onde $n_{e}$ é a freqüência do pico espectral, medida em Hertz, associado ao subintervalo dos turbilhões que mais contém energia cinética, $\sigma^{2}(z, t)$ é a variância da velocidade turbulenta calculada de $k_{e}$ à infinito,

$$
\frac{1}{2} \sigma^{2}(z, t)=\int_{k_{e}}^{\infty} \mathbf{E}(k, z, t) d k
$$

Para determinarmos a variância da velocidade e o coeficiente de difusão é necessário calcular o coeficiente de viscosidade cinemático $v_{T}$. Este coeficiente pode ser determinado pela equação (5) com a variância da velocidade dada pela integral do espectro de energia no subintervalo inercial derivado por Kaimal (1976), desde $n_{I}$ até o infinito.

$$
v_{T}=\frac{\beta}{6} \frac{1}{n_{I}} \int_{n_{I}}^{\infty} \mathbf{S}_{I}(n) d n
$$

onde

$$
\mathbf{S}_{I}(n)=\frac{\alpha}{(2 \pi)^{2 / 3}} U^{2 / 3}\left(\frac{\psi_{\varepsilon}}{h}\right)^{2 / 3} w_{*}^{2} n^{-5 / 3}
$$

Substituindo (31) em (30) e considerando $n_{I} \approx 10 U / h$ (Kaimal et al, 1976) e $\beta=0,55 U / \sigma$ (Hanna, 1982; Degrazia e Anfossi, 1998), obtemos a seguinte expressão para o coeficiente de viscosidade cinemático,

$$
v_{T}=\left(\frac{1}{10}\right)^{2 / 3} \psi^{1 / 3} w_{*} h
$$

74 Rev. Ciência e Natura, Dispersion Process: 63 - 78 , 2000. 
A figura abaixo compara a energia cinética turbulenta, obtida com a variância da velocidade dada pela equação (29), o espectro 3-D dado pela equação (23), e o coeficiente de viscosidade dado por (32), com o resultado do modelo de LES de Nieuwstadt (Nieuwstadt et al, 1986) e com um modelo proposto por Nieuwstadt no mesmo artigo.

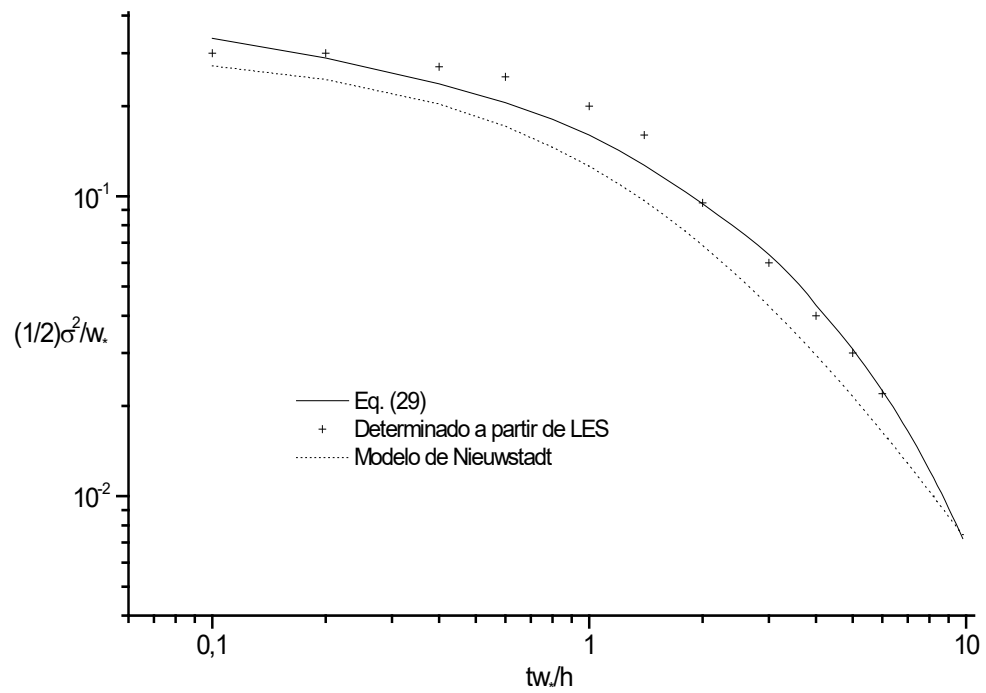

Fig.2 Evolução em função do tempo adimensional da energia cinética turbulenta

Pela Fig. 2 podemos observar que o modelo proposto neste trabalho pode ser utilizado para descrever o comportamento da energia cinética durante o decaimento da turbulência convectiva.

O coeficiente de difusão na Camada Residual pode ser estimado pela equação (28), com a variância da velocidade dada pela equação (29) e o espectro de energia calculado pela equação (23). O começo do subintervalo dos turbilhões que contém energia é considerado como o máximo do espectro dado pela equação (23).

Rev. Ciência e Natura, Dispersion Process: 63 - 78 , 2000. 


\section{CONCLUSÕES}

Neste trabalho é considerado o problema da derivação de uma parametrização para o coeficiente de difusão no decaimento da turbulência. Nesta derivação é utilizada uma equação dinâmica para a função espectro de energia, a teoria de difusão estatística clássica e a teoria de Heisenberg's para o decaimento da turbulência. Uma das principais suposições está relacionada com a identificação de uma freqüência, associada ao subintervalo inercial, que caracteriza a transferência inercial de energia entre os turbilhões de diferentes tamanhos. O coeficiente de difusão turbulento calculado, e representando o transporte turbulento durante o decaimento na CLC, pode ser empregado em problemas que envolvem a parametrização do transporte turbulento na $\mathrm{CR}$. Além do interesse científico intrínseco, este tema é relevante na descrição do transporte em mesoescala e simulações de difusão.

\section{AGRADECIMENTOS}

Trabalho parcialmente financiado pelo Conselho Nacional de Desenvolvimento Científico e Tecnológico (CNPq) e pela Fundação de Amparo a Pesquisa do Rio Grande do Sul (FAPERGS). 


\section{BIBLIOGRAFIA}

Batchelor, G.K.: 1949, 'The role of big eddies In homogeneous turbulence', Proc. Roy. Soc. A195, 513-532

Batchelor, O.K.: 1959, 'The theory of homogeneous turbulence', monografia de Mecânica e Matemática Aplicada, Cambridge University Press, 197 pp

Degrazia G.A. e Moraes O.L.L.: 1992, 'A model for eddy diffusivity in a stable boundary layer'. Boundary-Layer Meteorol, 58, 205-214

Degrazia G., Anfossi D., Moraes O.L.L. e Trini Castelli S.: 1997, 'A model for the turbulence parameterization in the residual layer'. AIR POLLUTION V, H. Power, T. Tirabassi e C.A. Brebbia editors, 101-107

Degrazia G.A., Anfossi D., Fraga De Campos Velho H. e Ferrero E.: 1998 'A Lagrangian decorrelation time scale for non-homogeneous turbulence' Boundary-Layer Meteorol., 86, 525-534

Degrazia, G.A. and Anfossi, D.: 1998, 'Estimation of the Kolmogorov constant $\mathrm{C}_{\mathrm{o}}$ from classical statistical diffusion theory', Atmos. Environm., 32, 36113614

Hanna S.R: 1981 'Lagrangian and Eulerian time-scale i the daytime boundary layer'. J. Appl. Meteor., 20, 242-249

Hinze J.O.: 1975, 'Turbulence', Mc Graw Hill, 790 pp

Kaimal J.C. Wyngaard J.C., Izumi Y. e Cote' O.R.: 1972, 'Spectral characteristics of surface layer turbulence' Quart. J.C. Roy. Meteorol. Soc., 98, 563-589

Kim, J. e Mahrt L.: 1992, 'Simple formulation of turbulent mixing in the stable free atmosphere and nocturnal boundary layer' Tellus 44A, 381-394

Kristensen, L., Lenschow, D., Kirkegaard, P. e Courtney, M.: 1989, 'The Spectral Velocity Tensor For Homogeneous Boundary-Layer Turbulence', Boundary-Layer Meteorol. 47,149-193

Moeng, C. H. e Sullivan P. P.: 1994, 'A Comparison of Shear- and BuoyancyDriven Planetary Boundary Layer Flows', J. Atmos. Sci., 51, 999-1022. 
Nieuwstadt, F.T.M. e Brost R.A.: 1986, 'The decay of convective turbulence', J. Atmos. Sci. 43, 532-546

Pao, Y.H.: 1965, 'Structure of Turbulent Velocity and Scalar Fields at Large Wavenumbers', The Physics of Fluids, 8, 1063-1075

Stanisic, M.M.: 1988, 'The mathematical theory of turbulence', Berlin Nova York,501pp

Stull, L.B.: 1988, 'An introduction to Boundary Layer Meteorology', Kluwer Academic Publishers, Boston, 666 pp.

78 Rev. Ciência e Natura, Dispersion Process: 63 - 78 , 2000. 